\title{
Programmable Time-Multiplexed Switched-Capacitor Variable Equalizer for Arbitrary Frequency Response Realizations
}

\author{
R. Pérez-Aloe, J. F. Duque-Carrillo, E. Sánchez-Sinencio, J. M. Valverde, G. Torelli, A. H. Reyes, and F. Maloberti
}

\begin{abstract}
A time-multiplexed digitally-programmable switched-capacitor (SC) variable equalizer which allows the realization of arbitrary frequency responses is presented. The circuit performs the same operation as a cascade of $N$ secondorder programmable equalizers, where $N$ is also the multiplexing order. Except the storing capacitors, the rest of the circuitry is shared for all individual equalizer functions (channels), resulting in silicon area savings higher than $60 \%$ with respect to a direct circuit implementation for $N=4$. The impact on circuit performance of crosstalk effects is discussed. Experimental results of a 3-V timesharing $\mathrm{SC}$ equalizer architecture fabricated in a CMOS $1.2 \mu \mathrm{m}$ technology are given for different values of the multiplexing order. The circuit has been designed to be incorporated in a programmable hearing aid device.
\end{abstract}

Index Terms - MOS analog integrated circuits, programmable filters, sampled data filters, switched capacitor filters, time division multiplexing.

\section{INTRODUCTION}

$\mathbf{S}$ WITCHED-CAPACITOR (SC) filtering is a very well established technique to process signals mainly in the audio frequency range. Advantages such as the insensitivity of the response to the unavoidable process fabrication deviations, as well as the possibility of having a digital control over their characteristics, constitute two of the stronger points of these kinds of circuits.

Today there is an increasing demand for circuits able to meet low power consumption and low silicon area requirements, especially for portable battery-operated systems with room constraints. In some systems, even area and power savings can become more important design targets than an extremely high performance, although the loss in the performance has not been excessive. Conventional design approaches of SC filters require a number of amplifiers which are proportional to the order of the realized transfer function and, in the case of high-order programmable SC filters, can be very expensive in terms of silicon area due to the capacitor banks that control the response. Because of their ability to relax power and area requirements, considerable attention has been paid to timesharing or multiplexing techniques for the implementation of either high-order SC filters [1]-[7] or SC filter banks [8],

Manuscript received March 3, 1996; revised July 10, 1996. This work was supported in part by the Spanish R\&D Plan (Grant TIC94-0616) and Iberdrola S.A. (Spain).

R. Pérez-Aloe, J. F. Duque-Carrillo, and J. M. Valverde are with the Department of Electronic and Electromechanical Engineering, University of Extremadura, (06071) Badajoz, Spain.

E. Sánchez-Sinencio and A. H. Reyes are with the Department of Electrical Engineering, Texas A\&M University, College Station, TX 77843, USA.

G. Torelli and F. Maloberti are with the Department of Electronics, University of Pavia, 27100 Pavia, Italy.

Publisher Item Identifier S 0018-9200(97)01351-6.
[9]. Some of the proposed timesharing schemes for high-order filters [1]-[4] use a single amplifier per pole pair, achieving a reduction by a factor of two in the amplifier count. Other proposed multiplexing circuits [5] reduce by a factor of $N$ the number of amplifiers required to implement an arbitrary $N$ thorder filter function using state space synthesis techniques, although some storage element is needed to perform multiplexing. Other interesting timesharing approaches implement a cascade of biquadratic SC cells with just two op-amps [6] or by sequentially computing the individual outputs of the cascade [7].

In this work, prompted by the necessity of having available an economical structure in terms of area and power and, simultaneously, a high degree of flexibility to implement any arbitrary frequency response with reasonable accuracy, a timemultiplexed scheme of a digitally-programmable SC bump equalizer is introduced. The time-shared circuit performs as $N$ series-connected second-order programmable equalizers, where $N$ is also the multiplexing order. The responses of the individual equalizers are computed sequentially and the only set of capacitor arrays required is dynamically reconfigurated according to the programming bits stored in a memory register.

\section{Programmable Time-Multiplexed SC EQUALIZER ARCHITECTURE}

Any frequency response can be realized with a cascade of $N$ second-order programmable magnitude equalizers $\left(\mathrm{BE}_{i}\right)$, as illustrated in the block diagram of Fig. 1, provided that $N$ is large enough. The general equalizer structure is programmable in the central frequency $\left(f_{o i}\right)$, gain $\left(A_{i}\right)$ at the central frequency and bandwidth $\left(\mathrm{BW}_{i}\right)$ of each section. The complete transfer function of the cascade will be given by the product of the $N$ individual second-order equalizer transfer functions [10], as the following expression indicates

$$
H(s)=\frac{V_{\text {out }}(s)}{V_{\text {in }}(s)}=\prod_{i=1}^{N} \frac{s^{2}+A_{i} \mathrm{BW}_{i} s+\omega_{o i}^{2}}{s^{2}+\mathrm{BW}_{i} s+\omega_{o i}^{2}}
$$

where the bandwidth is defined as $\mathrm{BW}_{i}=\omega_{o i} / Q_{i}$. Obviously, the higher the flexibility required to fit more precisely any frequency response shape is, the larger the number of cascaded second-order sections is, and therefore, power and area consumptions become progressively more unacceptable for micropower systems with room constraints.

The sampled-data nature of SC circuits, as well as the possibility of being programmable with a digital word, can be exploited to implement the transfer function of (1) in a time-multiplexed way. Fig. 2 shows the conceptual diagram of the time-multiplexed system along with the associated 


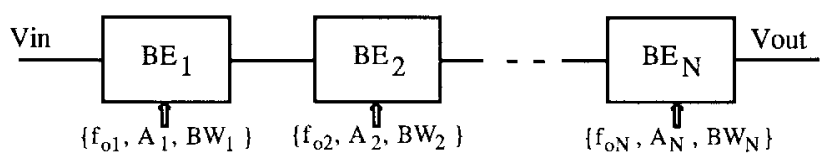

Fig. 1. A cascade of $N$ second-order programmable variable equalizers.

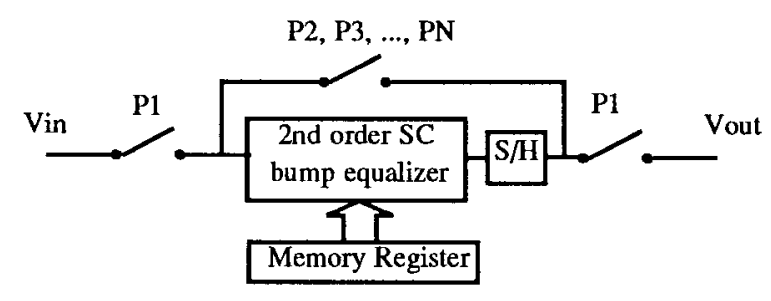

(a)

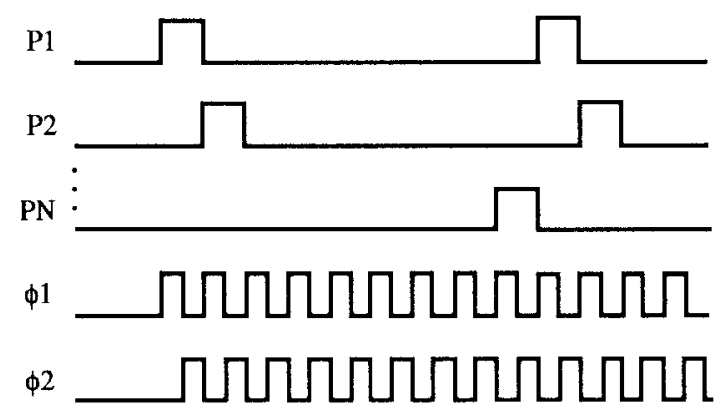

(b)

Fig. 2. (a) Block diagram of the time-multiplexed system and (b) clock waveforms.

clock waveforms. The bump equalizer section works with nonoverlapping clock phases $\phi_{1}$ and $\phi_{2}$. The operation of the system is as follows. At the clock period $P_{1}$, the input signal is sampled and the multiplexed circuit is configured to act as the first equalizer $\mathrm{BE}_{1}$. The output of $\mathrm{BE}_{1}$ is computed, stored, and fed back to the cell input in the next clock period $P_{2}$. At the beginning of this period $P_{2}$, the time-multiplexed equalizer is configurated to perform the operation of $\mathrm{BE}_{2}$. The capacitor banks are dynamically reprogrammed according to the corresponding memory registers contents during the small delays existing between the rising edges of the clock pulses $P_{i}$ and $\phi_{1}$. The successive equalizer responses are computed sequentially and, at the end of the next clock period $P_{1}$, the output sample corresponding to the complete equalization cascade is available, achieving a considerable silicon area saving with respect to the nonmultiplexed scheme shown in Fig. 1. The fact that an output sample is available at the end of next clock period $P_{1}$ depends, of course, on the particular type of processing carried out in the bump equalizer and sample-and-hold $(\mathrm{S} / \mathrm{H})$ circuits, as will be shown below.

In regard to power consumption, it is clear that the proposed multiplexed solution of Fig. 2 divides by $N$ the number of amplifiers that otherwise would be required. However, the effective clock period available for slewing plus settling is also divided by $N$ and, hence, the amplifiers have to be designed with higher performance (larger gain-bandwidth product and slew-rate) if the same transfer charge accuracy is to be maintained. As a consequence, larger current consumption and transistor aspect ratios are required with respect to a nonmultiplexed solution and, therefore, we can conclude that amplifier power and area consumptions are not so greatly reduced as the remaining circuitry area.

Fig. 3 shows the circuit schematic of our time-multiplexed second-order SC bump equalizer. Every capacitor array enclosed in a square box indicates a capacitor array that is dynamically reconfigurated in the way explained above. The multiplexed circuit has been designed to perform, at most, with a multiplexing order of four, but the extension of the operation to higher multiplexing orders does not introduce any conceptual difference. Notice that the architecture of Fig. 3 uses a fully differential (FD) topology but, for simplicity reasons, one half of the circuitry, as well as the switches that disconnect the capacitors $C_{A}$ and $C_{O i}$ from the virtual ground amplifiers terminals when they are inactive, have been omitted. In the same circuit schematic of Fig. 3, the basic SC bump equalizer architecture [11] is highlighted with a thicker line. The bump equalizer incorporates an SC integrator with low area requirements [12], although other SC integrator structures can be used as well. The operation principle of this SC integrator consists of taking advantage of the integrator idle phase to attenuate the signal so that the time-constant is determined by the product of two capacitor ratios. The capacitance spread is reduced by a factor of $B$, where $B$ equals $1+C_{D} / C_{A}$. The $z$-domain equalizer transfer function is given by the following expression:

$$
\begin{aligned}
H(z) & =\frac{V_{\text {out }}^{o}(z)}{V_{\text {in }}^{o}(z)} \\
& =-\frac{z^{2}+z\left(\frac{C_{w}^{2}}{C_{o}^{2}} \frac{1}{B^{2}}+\frac{C_{Q} C_{k}}{C_{s} C_{o}} \frac{1}{B}-2\right)+\left(1-\frac{C_{Q} C_{k}}{C_{s} C_{o}} \frac{1}{B}\right)}{z^{2}+z\left(\frac{C_{w}^{2}}{C_{o}^{2}} \frac{1}{B^{2}}+\frac{C_{Q}}{C_{o}} \frac{1}{B}-2\right)+\left(1-\frac{C_{Q}}{C_{o}} \frac{1}{B}\right)} .
\end{aligned}
$$

The equalizer parameters $f_{o} \cong\left(C_{\omega} f_{c}\right) /\left(2 \pi C_{o} B\right), A=$ $C_{k} / C_{s}, \mathrm{BW} \cong\left(C_{Q} f_{c}\right) /\left(2 \pi C_{o} B\right)$, where $f_{c}$ represents the sampling frequency, can be independently programmed by means of the capacitor arrays $C_{\omega}, C_{k}$, and $C_{Q}$, respectively. An outstanding feature of the basic SC bump equalizer used from the time-multiplexing point of view consists in that every programming capacitor bank in the integrators corresponds to an input capacitor rather than an integrating capacitor. Thus, any capacitor bank can be reconfigurated from one channel to another without any loss of information, since the output voltage samples of each integrator are stored in the feedback capacitors until the next clock period $P_{i}$.

The $\mathrm{S} / \mathrm{H}$ cell used in the time-multiplexed circuit corresponds to the structure reported in [13]. This FD S/H circuit samples the equalizer output during $\phi_{1}$, holds the signal during $\phi_{2}$, and either feeds it back to the cell input at the next phase $\phi_{1}$ if the clock period $P_{i}$ is different from $P_{1}$, or provides a valid output sample otherwise.

Since in the general second-order variable equalizer structure (see highlighted portion of Fig. 3), the input signal is injected into three different nodes, corresponding to the lowpass (LP), band-pass (BP) and high-pass (HP) paths, some of these basic functions can be obtained in a particular clock period $P_{i}$ with the general multiplexed scheme of Fig. 3 by 


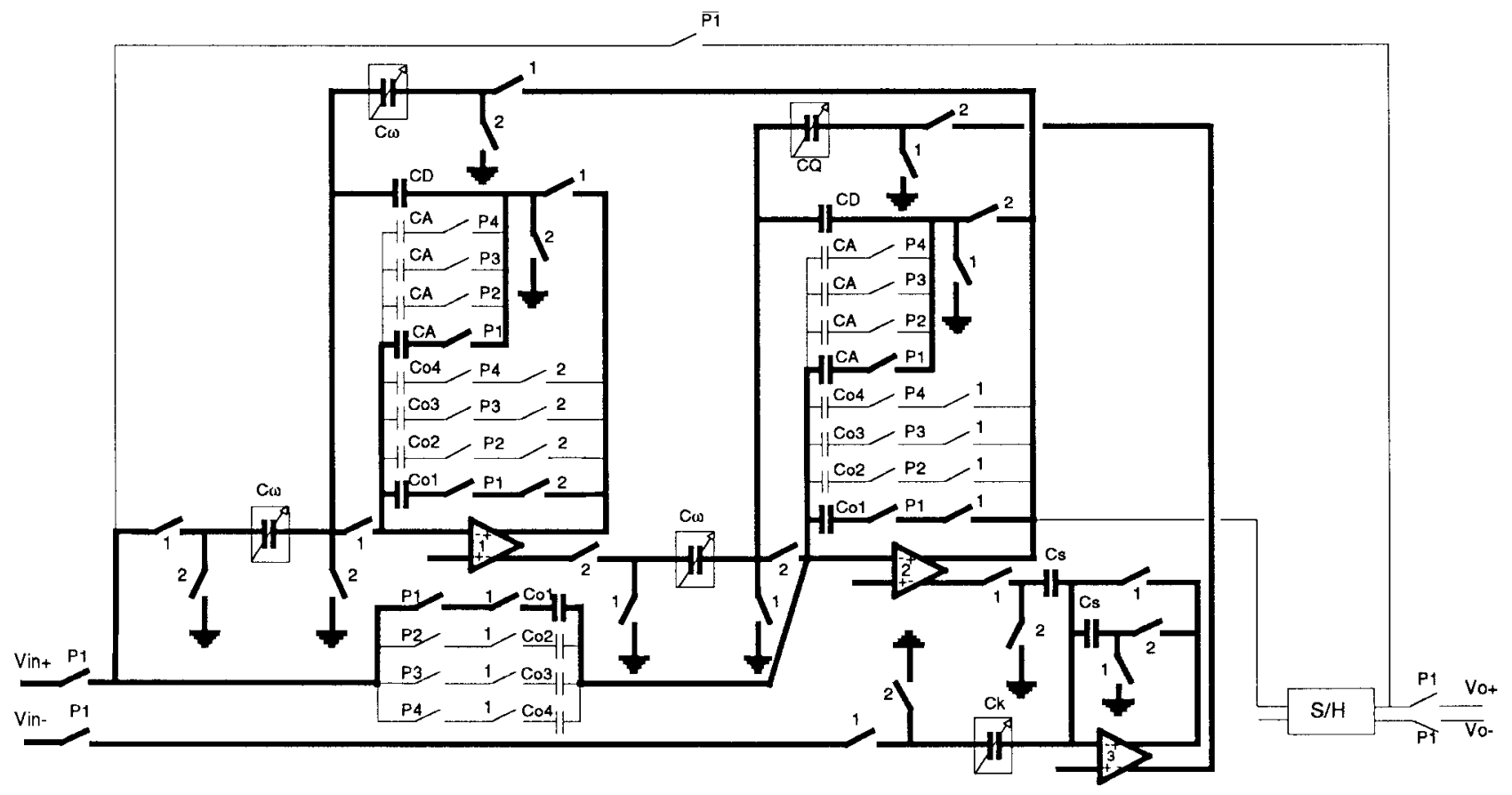

Fig. 3. Time-multiplexed digitally programmable SC variable equalizer with maximum multiplexing order of four.

proper values of the programming bits. Thus, to perform the operation of an HP filter, it is enough to program the capacitor array $C_{\omega}$ connected to the input and the array $C_{k}$ for zero capacitance value during the corresponding period $P_{i}$. However, in order to have this possibility available through the programming bits, the corresponding capacitor arrays must be implemented without any fixed capacitor in the bank, which reduces the maximum area savings achievable. In our design, the circuit of Fig. 3 can perform the operation of an HP filter followed by a cascade of three bump equalizers. The HP filter operation is achieved by eliminating the corresponding signal paths during $P_{1}$ by means of two additional switches not shown in the general structure of Fig. 3.

As pointed out above, the SC integrator used in the equalizer reduces the capacitance spread by a factor of $B$, however, the input-referred amplifier offset voltage is increased by the same amount. It can be demonstrated [11] that the total dc offset at the equalizer output $\left(V_{\text {os }}\right)$ is only influenced by the offset voltage ( $\left.V_{\text {off1 }}\right)$ of amplifier 1 . The reason why the offset voltages of amplifiers 2 and 3 do not contribute to the total offset voltage can be found in the fact that the resulting transfer functions from the inputs of these amplifiers to the output correspond to high-pass and band-pass responses, respectively, and therefore, are eliminated for $z=1(\omega=0)$. In the case of the time-multiplexed implementation, this dc voltage is propagated and added to the different individual equalizer contributions. Thus, the general expression which provides the output dc offset as a function of the multiplexing order can be easily derived, obtaining

$$
V_{\mathrm{os}}=\sum_{i=1}^{N}(-1)^{N+1}\left(2+\frac{C_{D}}{C_{\omega, p_{i}}}\right) V_{\mathrm{off} 1}
$$

where $C_{\omega, p i}$ accounts for the capacitance value $C_{\omega}$ at the generic clock period $P_{i}$. The exponential term $(-1)^{N+1}$ arises as a consequence of the sign inversion that takes places in the equalizer transfer function of (2). A tradeoff solution has been adopted between reduction in capacitive spread and increase in offset voltage.

\section{CROsstalk EfFects}

There are several potential mechanisms associated with the operation of the time-shared circuit of Fig. 3 which can lead to some loss of accuracy. Some of these effects arise from the leakage currents that discharge the hold capacitors of the $\mathrm{S} / \mathrm{H}$ circuit, as well as the integrating capacitors, while the corresponding channels are inactive. The use of large capacitor values and minimum sized transistor switches reduces the leakage effect, however, the resulting time constants are limited by the sampling period available.

Nonetheless, the more important cause of loss of accuracy is due to the crosstalk mechanisms among channels originated by some parasitic capacitances that couple the individual equalizer operations. The crosstalk effect caused by the parasitic capacitances associated with the virtual ground nodes of the amplifiers can be neglected if the open-loop gain is designed large enough. However, the strongest impact is introduced by the parasitic capacitance $C_{p}$ that couples input and output amplifier terminals as Fig. 4 shows. This capacitance causes some charge loss at the instants when the integrating capacitors $C_{o i}$ are connected, since the stored charge must be shared with the parasitic capacitance, reducing the amplifier output voltage. Fig. 5 illustrates the impact on the circuit of Fig. 3 of the crosstalk effect for different values of $C_{p}$. As can be observed, the simulated operation of the time-multiplexed equalizer is an HP filter cascaded with a variable equalizer, which means a multiplexing order of two. With a proper circuit layout, this crosstalk capacitance can be kept to a minimum 


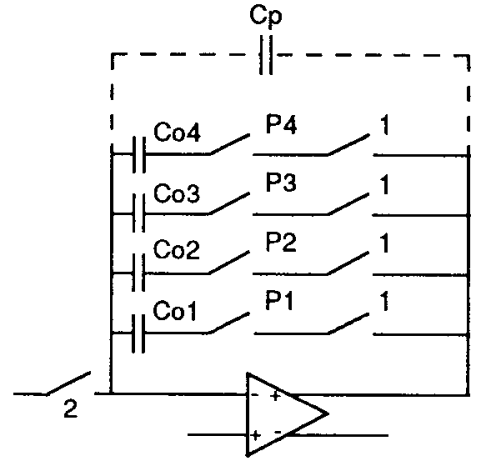

Fig. 4. A time-shared SC integrator showing the crosstalk parasitic capacitance $C_{p}$.

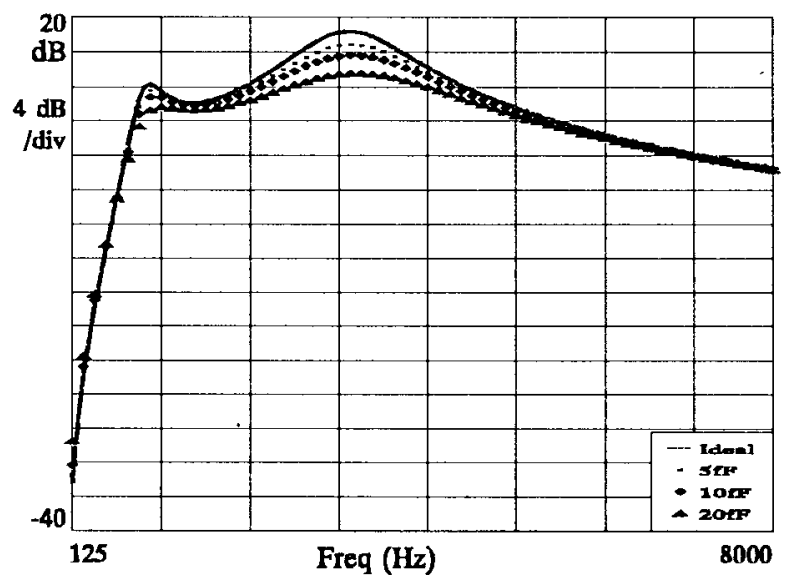

Fig. 5. Simulated results illustrating the crosstalk effect for different parasitic capacitance values $C_{p}(N=2)$.

value. Moreover, in [4], a design solution has been proposed for partial compensation of this crosstalk effect in FD SC circuits, but it requires an extra clock phase and slightly more complicated hardware.

\section{EXPERIMENTAL RESULTS}

The programmable time-multiplexed bump equalizer has been designed and fabricated in a 1.2- $\mu \mathrm{m}$ n-well double-poly double-metal CMOS process (Fig. 6). The circuit occupies $2.7 \times 1.3 \mathrm{~mm}^{2}$, including some test blocks that allow the possibility of observing the different channel responses separately. It has been designed to operate with $3 \mathrm{~V}$ of total supply voltage and $50 \mathrm{kHz}$ of effective sampling frequency $\left(\phi_{1}, \phi_{2}\right)$ per channel. The amplifier incorporated consists of an FD folded-cascode structure with an internal feedback loop based on resistive degeneration of current mirrors [15] to carry out the required output common-mode component stabilization. The capacitor banks have been implemented as 3-b binary-weighted arrays. Therefore, any equalization channel can provide up to 512 different frequency responses, and the number of possible responses of the time-multiplexed equalizer, for $N=4$, results almost unlimited. The silicon area savings achieved is around $60 \%$ with respect to the nonmultiplexed implementation.

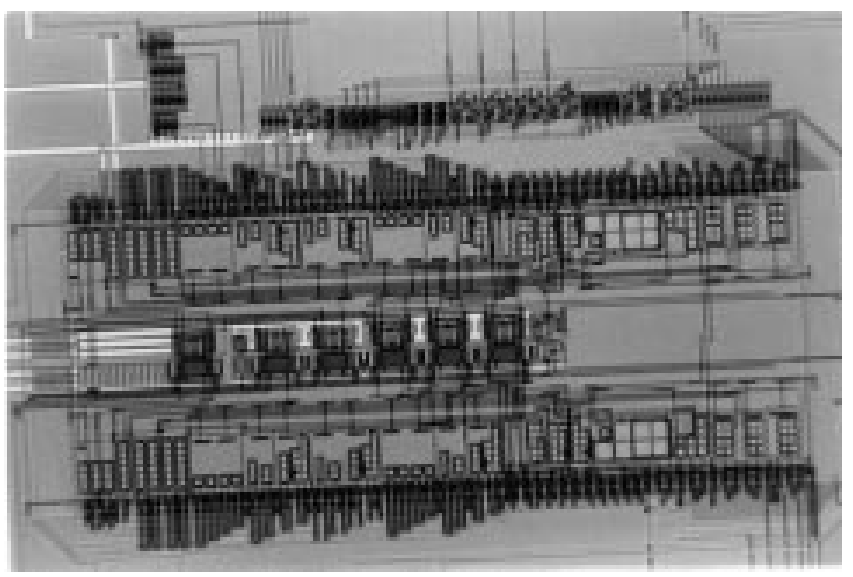

Fig. 6. Chip photomicrograph.

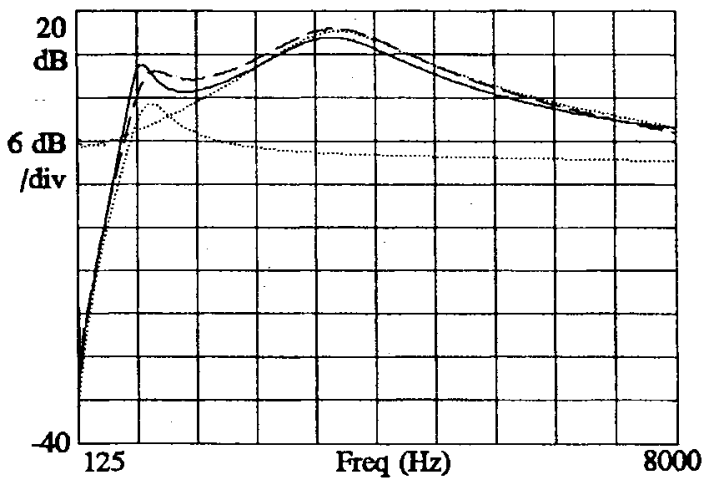

Fig. 7. An experimental circuit response $(N=2)$ equivalent to an HP filter followed by an equalizer (dotted curves: measured individual channel responses, dashed curve: ideal product of channel responses, solid curve: measured response of the complete circuit).

Fig. 7 shows an experimental frequency response of the time-multiplexed equalizer operating with a multiplexing order of two, which corresponds to the simulated response of Fig. 5. In the same plot, the measured individual responses of both channels (dotted curves) along with the ideal product of them (dashed curve) are also shown. We have estimated the total parasitic capacitance $C_{p}$ in our design, resulting in a value approximately equal to $2.4 \mathrm{fF}$. As observed in Fig. 7, the maximum deviation (approximately $2 \mathrm{~dB}$ ) between the ideal and the experimental multiplexed (solid curve) responses agrees well with the simulated results shown in Fig. 5, confirming this crosstalk mechanism as the main error source in the time-multiplexed scheme.

The current design has been carried out to be incorporated in a programmable hearing aid device as the cell responsible for frequency response correction. Thus, the resulting hearing aid circuit shows an almost unlimited capability for precise hearing loss compensation regardless of shape and, simultaneously, a very economical implementation mainly in terms of silicon area. For this hearing loss compensation purpose, we found that an appropriate time-multiplexed circuit operation consists of programming the system to act as a cascade composed by an HP filter followed by three series-connected variable equalizers as indicated above. Every channel of the timeshared circuit sweeps well-defined frequency range within the 


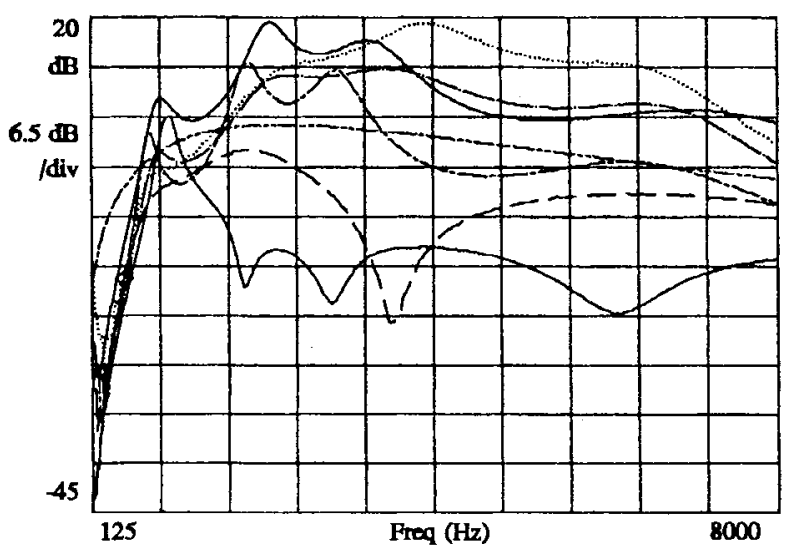

Fig. 8. Selected measured frequency responses with a multiplexing order of four.

TABLE I

Programmability Ranges of Different Parameters

\begin{tabular}{|c|c|c|c|}
\cline { 2 - 4 } \multicolumn{1}{c|}{} & $\mathrm{f}_{0}(\mathrm{kHz})$ & $\mathrm{A}_{0}(\mathrm{~dB})$ & $\mathrm{BW}(\mathrm{kHz})$ \\
\hline $\mathrm{HP}$ & $(.1,1)$ & $(0,18)$ & $(.125,1.5)$ \\
\hline $\mathrm{BE}_{1}$ & $(0.25,2)$ & $(-14,20)$ & $(0.25,3.5)$ \\
\hline $\mathrm{BE}_{2}$ & $(0.5,3)$ & $(-14,20)$ & $(0.5,7)$ \\
\hline $\mathrm{BE}_{3}$ & $(3,6.5)$ & $(-14,20)$ & $(3.5,15)$ \\
\hline
\end{tabular}

audio band of interest [125 Hz, $8 \mathrm{kHz}$. The programmability ranges of the different parameters are shown in Table I for $200 \mathrm{kHz}$ of total clock frequency. In order to illustrate the versatility of the time-multiplexed circuit, Fig. 8 shows several experimental frequency responses corresponding to different settings of programming bits. In practice, the programming bit setting for the best frequency response fitting of the time-multiplexed equalizer is determined with the help of a computer routine based on the downhill simplex method [16].

The total harmonic distortion (THD) measured for different amplitudes of a 1-kHz input sinewave is plotted in Fig. 9. THD remains below $1 \%$ up to $1.8-V_{\mathrm{pp}}$ differential input signal. For the distortion measurements, the individual equalizers were programmed with the flattest response which provides a total gain of approximately $4 \mathrm{~dB}$ at a frequency of $1 \mathrm{kHz}$. The inputreferred noise integrated over the frequency band $[125 \mathrm{~Hz}$, $8 \mathrm{kHz}]$ is $0.19 \mathrm{~m} V_{\mathrm{rms}}$. Combining these figures, a dynamic range higher than $70 \mathrm{~dB}$ results. The measured total current consumption is $62 \mu \mathrm{A}$.

\section{CONCLUSION}

A multiplexing technique for the realization of arbitrary frequency responses with very low silicon area requirements has been presented. The circuit consists of a time-shared SC magnitude-equalizer that performs the same operation as a cascade of digitally-programmable equalizers. The full programmability is achieved by means of only one set of capacitor arrays, which is dynamically reconfigurated according the programming bits. Crosstalk errors caused by input-output parasitic capacitances in the amplifiers are the main accuracy limitation of the technique. In our circuit, the maximum deviation between measured and ideal simulated responses was smaller than $2 \mathrm{~dB}$ for $N=2$. Experimental results obtained from a CMOS test-chip prototype with a maximum
THD (\%)

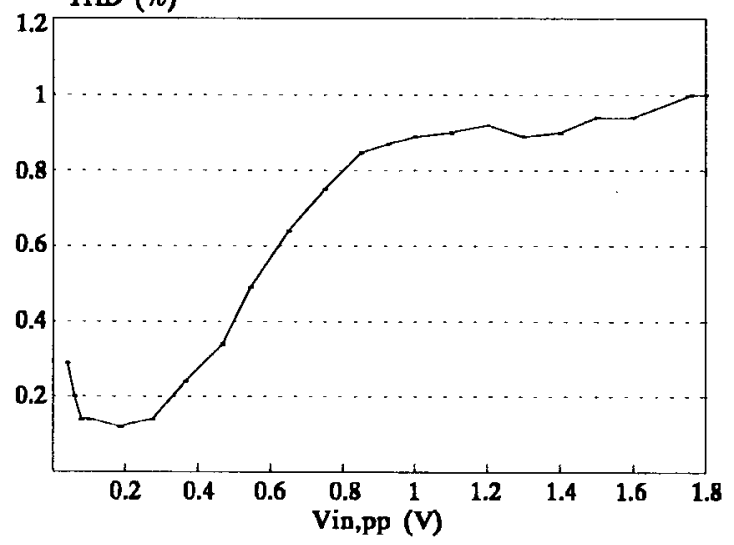

Fig. 9. THD for different input amplitude values $\left(f_{\text {in }}=1 \mathrm{kHz}\right)$.

multiplexing order of four have demonstrated the potentiality of the proposed circuit.

\section{REFERENCES}

[1] D. J. Allstot and K.-S. Tan, "Simplified MOS switched capacitor ladder filter structures," IEEE J. Solid-State Circuits, vol. 16, pp. 724-729, Dec. 1981.

[2] K. R. Laker, P. E. Fleischer, and A. Ganesan, "Parasitic insensitive, biphase switched capacitor filters realized with one operational amplifier per pole pair," Bell Syst. Tech. J., vol. 61, pp. 685-707, May-June 1982.

[3] P. V. Anada, V. Ramachandran, and M. N. S. Swami, "Parasitic compensated single amplifier SC biquad equivalent to Fleischer-Laker biquad," IEEE Trans. Circuits Syst., vol. 33, pp. 458-460, Apr. 1986.

[4] F. Montecchi, "Time-shared switched-capacitor ladder filters insensitive to parasitic effects," IEEE Trans. Circuits Syst., vol. 31, pp. 349-353, Apr. 1984.

[5] G. W. Roberts, W. M. Snelgrove, and A. S. Sedra, "Switched-capacitor realization of $N$ th-order transfer function using a single multiplexed op-amp," IEEE Trans. Circuits Syst., vol. 34, pp. 140-148, Feb. 1987.

[6] R. Castello, A. G. Grassi, and S. Donati, "A 500-nA sixth-order bandpass SC filter," IEEE J. Solid-State Circuits, vol. 25, pp. 669-676, June 1990.

[7] C.-Y. Wu, J.-C. Bor, and B. S. Jeng, "Realizations of high-order switched-capacitor filters using multiplexing technique," IEEE Trans. Circuits Syst.-II, vol. 41, pp. 778-785, Dec. 1994.

[8] J. S. Chang and Y. C. Tong, "A micropower-compatible timemultiplexed SC speech spectrum analyzer design," IEEE J. Solid-State Circuits, vol. 28, pp. 40-48, Jan. 1993.

[9] P. W. Bosshart, "A multiplexed switched-capacitor filter bank," IEEE J. Solid-State Circuits, vol. 15, pp. 939-945, Dec. 1981.

[10] J. F. Duque-Carrillo, J. Silva-Martínez, and E. Sánchez-Sinencio, "Programmable switched-capacitor bump equalizer architecture," IEEE $J$. Solid-State Circuits, vol. 25, pp. 1035-1039, Aug. 1990.

[11] J. F. Duque-Carrillo, P. Malcovati, F. Maloberti, R. Pérez-Aloe, A. H. Reyes, E. Sánchez-Sinencio, G. Torelli, and J. M. Valverde, "VERDI: An acoustically programmable and adjustable CMOS mixed-mode signal processor for hearing aid applications," IEEE J. Solid-State Circuits, vol. 31, pp. 634-645, May 1996.

[12] H. Qiuting, "A novel technique for the reduction of the capacitance spread in high- $Q$ SC circuits," IEEE Trans. Circuits Syst., vol. 36, pp. 121-126, Jan. 1989.

[13] G. Nicollini, P. Confalonieri, and D. Senderowicz, "A fully differential sample-and-hold circuit for high-speed applications," IEEE J. Solid-State Circuits, vol. 24, pp. 1461-1464, Oct. 1989.

[14] S. C. Fang, Y. Tsividis, and O. Wing, "SWITCAP: A switched-capacitor network analysis program (Parts I and II)," IEEE Circuits Syst. Mag., vol. 5, pp. 4-10, Sept.-Dec. 1983.

[15] J. F. Duque-Carrillo, "Control of the common-mode component in CMOS continuous-time fully-differential signal processing," Analog Integrated Circuits and Signal Processing. Boston, MA: Kluwer, vol. 4, pp. 131-140, Sept. 1993.

[16] W. H. Press, B. P. Flannery, S. T. Teukolsky, and W. T. Vetterling, $N u-$ merical Recipes: The Art of Scientific Computing, 3rd ed. Cambridge, MA: Cambridge, p. 289, 1988. 